\title{
A 6.5-GHz Cryogenic All-Pass Filter Circulator in 40-nm CMOS for Quantum Computing Applications
}

\author{
Andrea Ruffino ${ }^{\# 1}$, Yatao Peng ${ }^{\#}$, Fabio Sebastiano $\$$, Masoud Babaie ${ }^{\$}$, Edoardo Charbon ${ }^{\# 2}$ \\ \#École Polytechnique Fédérale de Lausanne, Switzerland \\ $\$$ Delft University of Technology, The Netherlands \\ 1andrea.ruffino@epfl.ch, ${ }^{2}$ edoardo.charbon@epfl.ch
}

\begin{abstract}
Cryogenic solid-state quantum processors require classical control and readout electronics; to achieve compactness and scalability, cryogenic integrated circuits have been recently proposed for this goal. Circulators are widely used in readout circuits, however they are typically discrete bulky devices, thus preventing miniaturization. To address this issue, we propose a fully integrated $40-\mathrm{nm}$ CMOS $6.5-\mathrm{GHz}$ circulator operating from $300 \mathrm{~K}$ to $4.2 \mathrm{~K}$. At $300 \mathrm{~K}$, it achieves a 2.2-dB insertion loss, an 18-dB isolation, and a 2.4-dB noise figure over the 1-dB bandwidth from $5.6 \mathrm{GHz}$ to $7.4 \mathrm{GHz}$, with a core power of only $2.5 \mathrm{~mW}$. This improves to $2.1 \mathrm{~mW}$ core power at $4.2 \mathrm{~K}$, while showing 1.3-dB insertion loss and 17-dB isolation over the 1-dB bandwidth from $5.8 \mathrm{GHz}$ to $7.6 \mathrm{GHz}$. The circuit achieves a record-low core power and a $1.6 \times$ wider fractional bandwidth than the state-of-the-art, thus allowing its use for multiple channels in power-constrained cryogenic refrigerators. These advances are enabled by a fully-passive architecture based on LC all-pass filters, allowing the use of a lower clock frequency than in prior art.
\end{abstract}

Keywords - Cryo-CMOS, circulator, qubit, spin qubit, superconducting qubit, qubit readout, quantum computing.

\section{INTRODUCTION}

Quantum computing is a promising solution to the everincreasing demand for computational power. State-of-the-art solid-state quantum processors, such as those based on spin quantum bits (qubits) [1] or on superconducting qubits [2], operate at deep-cryogenic temperature while the complex $\mathrm{RF}$ setup required for their control is implemented by offthe-shelf instrumentation at room temperature. Although this approach is feasible for the few qubits $(<100)$ available today, it will become unpractical to wire room-temperature electronics to the thousands of qubits required in practical quantum computers. CMOS circuits operating directly at cryogenic temperatures (cryo-CMOS) can pave the way for co-integration of qubits and classical control, so as to achieve compact and scalable systems in the near future [3].

Cryogenic circulators are commonly used in qubit readout systems, as shown in Fig. 1, however they are currently implemented with bulky ferrite devices, thus preventing system integration. Integrated circulators operating at room temperature have been recently proposed for full-duplex radio transceivers, exploiting time-varying circuits to achieve the circulator's non-reciprocal response. CMOS implementations of circulators have been demonstrated in the K-band, based on switched transmission lines [4], and in the GSM band, based on N-path filters [5], however neither approach is well suited for the $5-8 \mathrm{GHz}$ band required for superconducting qubits [2].
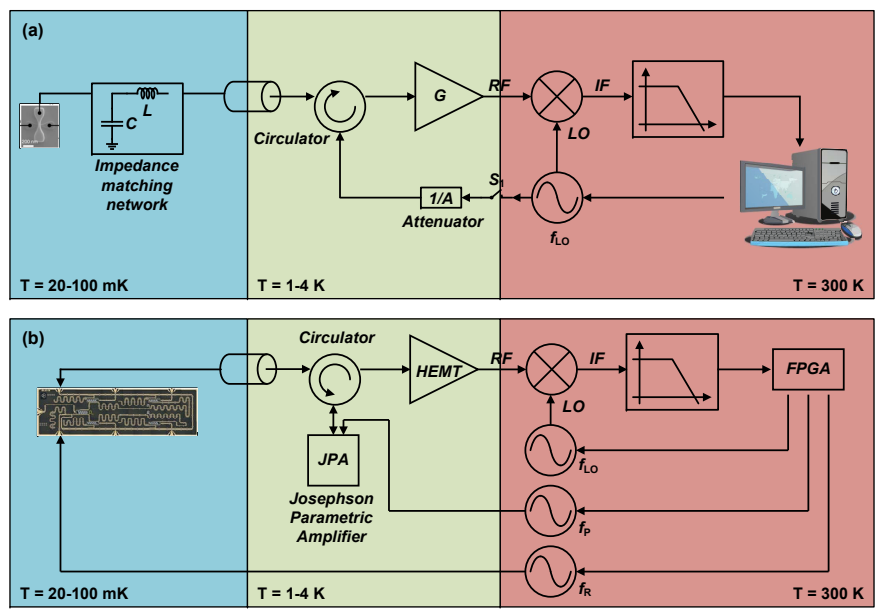

Fig. 1. Circulators used in readout circuits for (a) spin qubit processors [1] and (b) superconducting qubit processors [2].

Switched transmission lines require $\lambda / 4$ transmission lines at the switching frequency (i.e., $1 / 3$ of the $6.5-\mathrm{GHz}$ operating frequency), thus resulting in a significant penalty on the circulator's die area and insertion loss. N-path filters require non-overlapping clock phases at the $6.5-\mathrm{GHz}$ operating frequency, thus resulting in excessive power dissipated by the clock drivers. Minimizing power consumption is essential in cryogenic applications because of the very limited cooling power available in existing cryostats. Substantial power savings can be achieved by re-using the same electronics for multiple qubit channels via frequency multiplexing, thus requiring bandwidth maximization.

To address these issues, we propose the first fully integrated CMOS circulator operating at cryogenic temperatures. By exploiting a novel architecture based on all-pass filters, we avoid both large-area transmission lines and power-hungry high-frequency clock drivers. This results in a passive circulator operating down to $4.2 \mathrm{~K}$ with large bandwidth, while dissipating only $2.1 \mathrm{~mW}$. Target applications involve superconducting qubits (hence the operating frequency band), but do not exclude spin qubit experiments at $5-8 \mathrm{GHz}$.

\section{Design AND ARChitecture}

Existing non-magnetic CMOS circulators are formed by a loop with two reciprocal branches, providing $90^{\circ}$ reciprocal phase shifts, and a non-reciprocal branch, the core component, creating a non-reciprocal $0^{\circ} / 180^{\circ}$ phase shift depending on the signal direction, as shown in Fig. $2 \mathrm{a}$. 
(a)

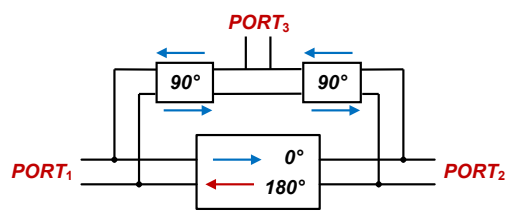

(b)

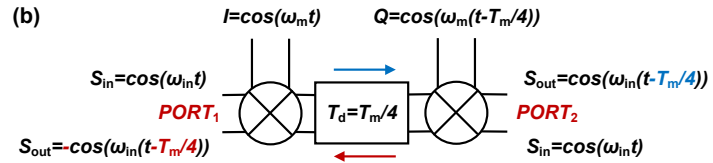

(c)

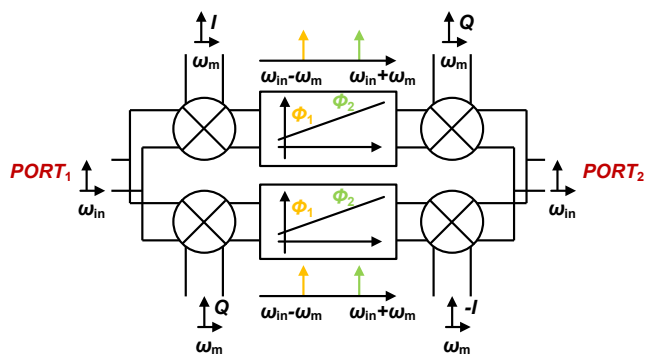

Fig. 2. Block diagram of (a) the circulator, including (b) the time domain analysis and (c) the frequency domain analysis of the non-reciprocal branch.

Both reciprocal and non-reciprocal branches create the required phase shifts using passive filters, which are implemented as CLC low-pass filter T sections [5], or lumpedelement Bragg-limited transmission line sections [4].

The non-reciprocal branch, shown in Fig. 2b, is realized by inserting a filter between two I/Q mixers switching at a frequency $\omega_{\mathrm{m}}$, while the incoming signal travels at frequency $\omega_{\text {in }}$, with $\omega_{\mathrm{m}}<\omega_{\text {in }}$ to enable low-power operation. In order to have a lossless transmission, the internal filter needs to produce a time delay $T_{d}$ equivalent to $T_{m} / 4$, where $T_{m}$ is the clock (LO) period [4]. In the forward direction, this yields a transmission with a delay of $\mathrm{T}_{\mathrm{m}} / 4$, while in the reverse direction this gives a delay of $\mathrm{T}_{\mathrm{m}} / 4$ and a sign flip. Time-variance introduced by mixers results in non-reciprocity.

In order to reduce the circulator power consumption, $\omega_{\mathrm{m}}$ must be reduced (or its period $\mathrm{T}_{\mathrm{m}}$ increased). This means that the time delay introduced by the internal filter needs to be larger. If this is implemented with transmission lines, then the corresponding line needs to be longer, which requires more equivalent LC sections. This would result in a larger insertion loss and area, or, if a smaller number of sections is used, reduced bandwidth.

If, however, one considers the non-reciprocal branch shown in Fig. $2 c$ in the phase-frequency domain, a signal travelling at $\omega_{\text {in }}$ is I/Q mixed with the clock at $\omega_{\mathrm{m}}$, thus generating two mixing products at $\omega_{\mathrm{L}}=\omega_{\text {in }}-\omega_{\mathrm{m}}$ and $\omega_{\mathrm{H}}=\omega_{\mathrm{in}}+\omega_{\mathrm{m}}$, with corresponding phase shifts $\phi_{1}=\left(\omega_{\mathrm{in}}-\omega_{\mathrm{m}}\right) \mathrm{T}_{\mathrm{d}}$ and $\phi_{2}=\left(\omega_{\mathrm{in}}+\omega_{\mathrm{m}}\right) \mathrm{T}_{\mathrm{d}}$, respectively. Those components mix with $\mathrm{Q} / \mathrm{I}$ clock signals and appear again at $\omega_{\text {in }}$ at the other port of the non-reciprocal branch [4]. If $\phi$ is the phase shift between the set of mixers, the conditions for non-reciprocal operation can be expressed as:

$$
\left\{\begin{array}{c}
\phi=-\pi / 2 \\
\phi_{1}-\phi_{2}=2 \phi \\
\phi_{1}=\left(\omega_{\text {in }}-\omega_{\mathrm{m}}\right) \mathrm{T}_{\mathrm{d}}=\pi / 2 .
\end{array}\right.
$$
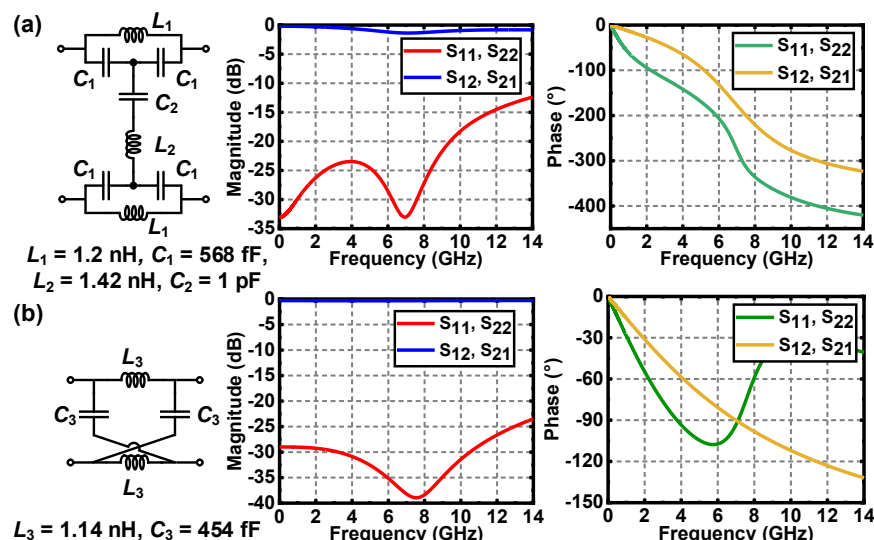

Fig. 3. Schematic and transfer function post-layout simulation of (a) a second order bridged-T all-pass filter and (b) a first order lattice all-pass filter.

The first equation sets the choice to have $90^{\circ}$ phase shift between clock signals, to yield a differential and I/Q clocking scheme and mitigate the effect of duty cycle mismatch [4].

The second equation describes the core functionality of the circulator and establishes that the phase shifts of $\omega_{\mathrm{L}}$ and $\omega_{\mathrm{H}}$ components need to be $180^{\circ}$ apart. If $\omega_{\mathrm{m}}$ is reduced to minimize power consumption, the $\omega_{\mathrm{L}}$ and $\omega_{\mathrm{H}}$ components get closer to one another, requiring a sharply dispersive filter.

Instead of employing first-order transmission line sections, we propose to use a second-order filter, namely a bridged-T LC all-pass filter, shown in Fig. 3a. In this case, the center frequency of the circulator can be placed at the center of the all-pass filter, where the phase shift is $-180^{\circ}$, while $\omega_{\mathrm{L}}$ and $\omega_{\mathrm{H}}$ are placed where the phase shift of the filter is $-90^{\circ}$ and $-270^{\circ}$ respectively. In such second-order all-pass filters, thanks to their sharp phase response, the second non-reciprocity condition is satisfied at frequencies closer to the center frequency. Consequently, a modulation index $\omega_{\text {in }} / \omega_{\mathrm{m}}=5$ can be achieved without transmission line trade-offs.

Such an approach allows to reduce clock frequency to minimize power consumption, while achieving area compactness, lower insertion loss and noise figure, since fewer inductors are in series with the signal path. The use of a second-order all-pass filter also allows to increase the circulator bandwidth thanks to the highly linear (as well as steep) phase response around the center frequency, capable of maintaining the required phase relationship for an extended frequency band. This is beneficial for frequency multiplexing, to accommodate multiple qubits on the same readout chain.

Finally, the third equation sets the insertion phase, which is chosen to be $90^{\circ}$, in order to eliminate the additional $45^{\circ}$ equivalent sections in [4], so as to further reduce the count of passives, with benefits on area and complexity.

We also propose to implement the reciprocal branches as LC all-pass filters, but in this case first-order lattice filters, shown in Fig. 3b, suffice to create $90^{\circ}$ phase shifts.

The non-reciprocal branch is thus resulting in an overall $0^{\circ} / 180^{\circ}$ non-reciprocal phase shift depending on the signal direction, and with the action of reciprocal branches, this yields a final constructive/destructive interference at all ports. The entire circulator is shown in Fig. 4. 


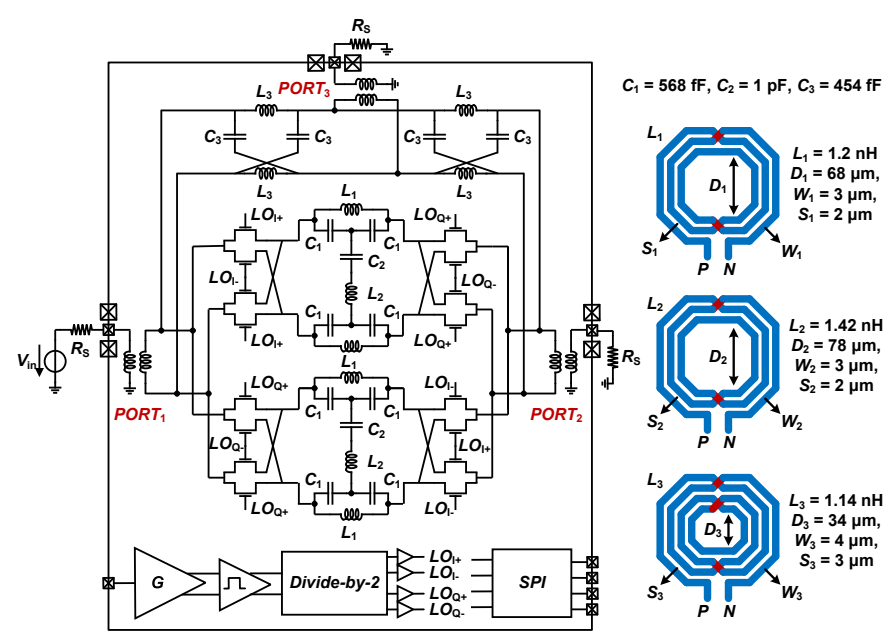

Fig. 4. Block diagram and circuit schematic of the designed circulator. Circulator core and auxiliary structures are shown.

\section{CHIP IMPLEMENTATION}

The proposed circulator was fabricated in 40-nm CMOS with an ultra-thick top metal layer, and the chip micrograph is shown in Fig. 5. Thanks to the all-pass filter implementation, the circulator occupies a core area of only $0.45 \mathrm{~mm}^{2}$. The LC filters have been realized with multi-turn spiral inductors and interdigitated MOM capacitors, for compactness and integration. Mixers have been realized as nMOS-only passive differential mixers with $\mathrm{W} / \mathrm{L}=50 \mu \mathrm{m} / 40 \mathrm{~nm}$, optimized to trade off $\mathrm{R}_{\mathrm{ON}}$ resistance, causing additional insertion loss, and parasitic capacitance $\mathrm{C}_{\mathrm{p}}$, producing unwanted phase shifts, which have been compensated for by filter design.

The system has been designed to operate at a center frequency of $6.5 \mathrm{GHz}$, with a clock signal at $1.3 \mathrm{GHz}$, thus yielding a modulation index $\mathrm{m}=6.5 \mathrm{GHz} / 1.3 \mathrm{GHz}=5$.

Transformer baluns have been used to convert on-chip differential signals to single-ended signals for RF probing on GSG pads and have been de-embedded from measurements.

The on-chip non-overlapping clock generator outputs the four required I/Q differential signals from a single external sinusoidal input. An active single-ended to differential amplifier at the input, cascaded by further gain stages, is used to clip the signal to a square wave, which is then fed to a $\mathrm{C}^{2} \mathrm{MOS}$ latch-based divider-by-2, finally reinforced by proper buffers and phase aligners. An on-chip SPI allows tuning of the amplifier bias and port impedance during testing.
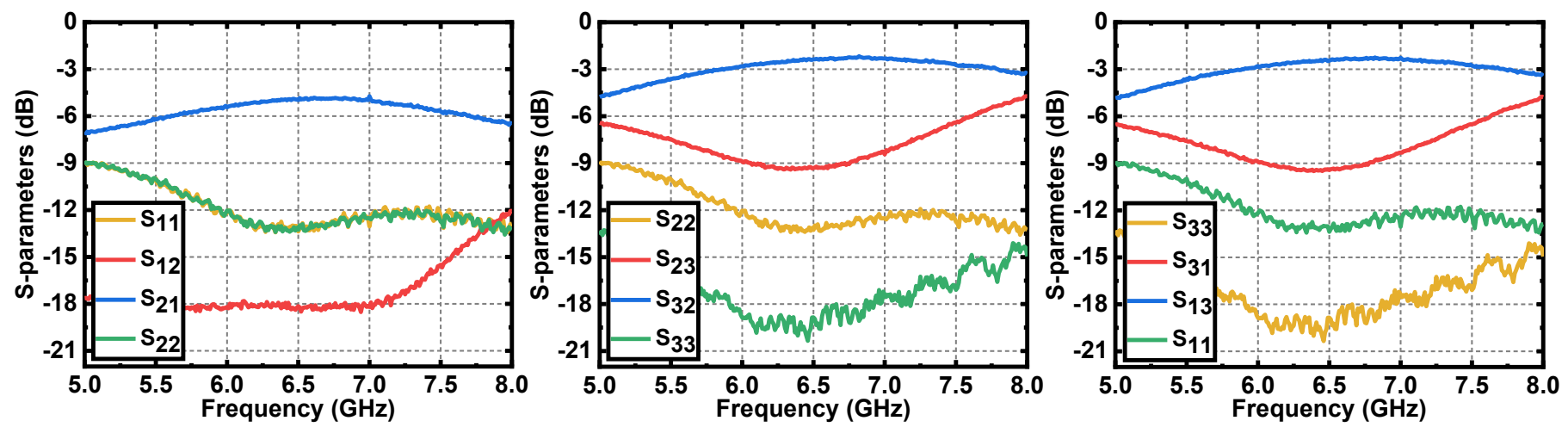

Fig. 6. Circulator S-parameter measurements at $300 \mathrm{~K}$.

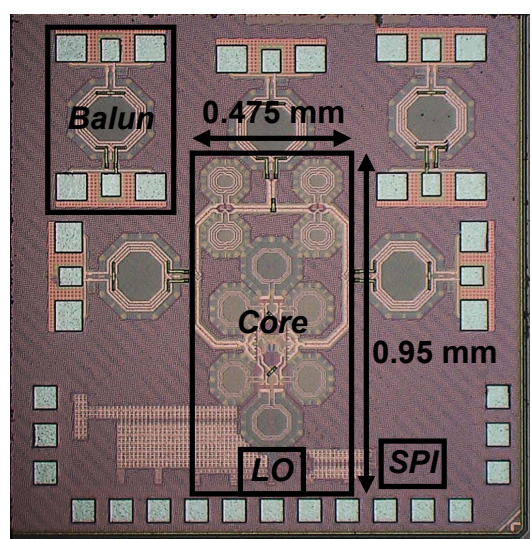

Fig. 5. Circulator chip micrograph, showing the circulator core, transformer

\section{Measurement Results And Discussion}

The fabricated prototype has been bonded to a PCB, while the GSG pads at the three ports have been probed with a Lake Shore CPX probe station at $300 \mathrm{~K}$ and $4.2 \mathrm{~K}$.

The DC power consumption of the circulator core (including frequency divider and buffers driving the mixers) is $2.3 \mathrm{~mA}$ from a $1.1 \mathrm{~V}$ power supply, while the auxiliary on-chip clock generation (amplifiers and phase aligners) consumes an additional $9 \mathrm{~mA}$ from a $1.1 \mathrm{~V}$ supply, when the internal clock signal is $1.3 \mathrm{GHz}$ (the external LO signal is $2.6 \mathrm{GHz}$ ). These values reduce to $1.9 \mathrm{~mA}$ and $7.7 \mathrm{~mA}$ respectively when the circuit is cooled down to $4.2 \mathrm{~K}$ under the same conditions.

The measured S-parameters at $300 \mathrm{~K}$, after calibration and balun de-embedding, are reported in Fig. 6. The circuit operates over a 1-dB insertion loss-isolation bandwidth of $5.6 \mathrm{GHz}-7.4 \mathrm{GHz}$, thus yielding a $28 \%$ fractional bandwidth, with a $2.2-\mathrm{dB}$ insertion loss and an $18-\mathrm{dB}$ isolation. Impedance matching at all ports is always below $-10 \mathrm{~dB}$. These results are in good agreement with post-layout simulations.

Fig. 7 shows the measured S-parameters at $4.2 \mathrm{~K}$. The $1-\mathrm{dB}$ operational bandwidth is $5.8 \mathrm{GHz}-7.6 \mathrm{GHz}$, the minimum insertion loss is reduced to $1.3 \mathrm{~dB}$, while the isolation becomes $17 \mathrm{~dB}$. This improvement at cryogenic temperatures can be explained by an increase in quality factor of passives, in particular inductors, due to the reduction of substrate losses thanks to carrier freeze-out, and by a reduction of $\mathrm{R}_{\mathrm{ON}}$ resistance of the mixer switches in series with the signal path, due to larger carrier mobility [3]. baluns, and auxiliary structures. 

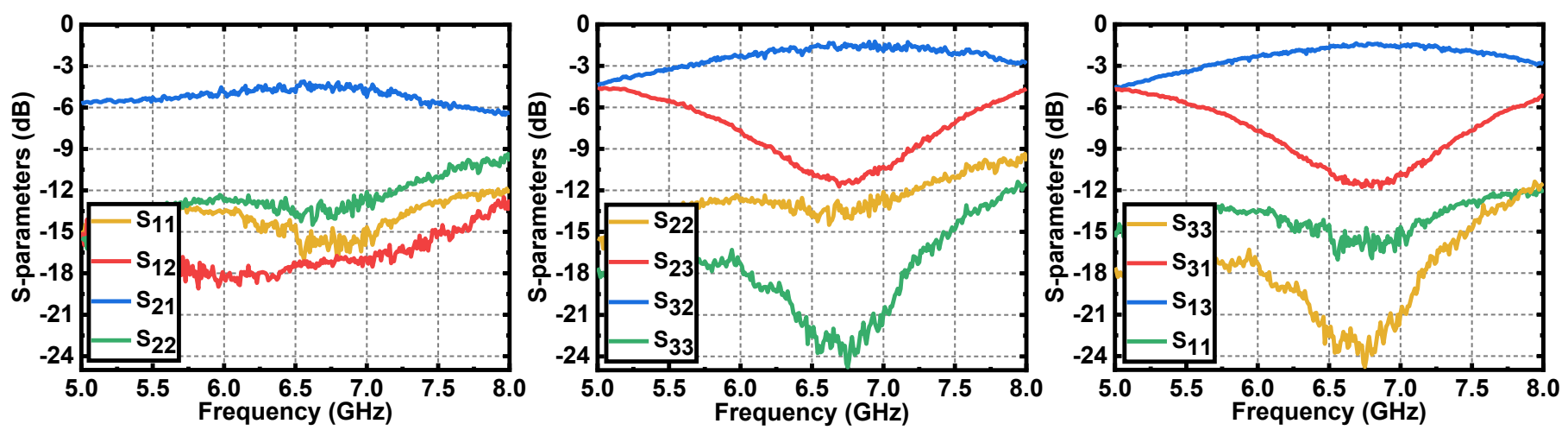

Fig. 7. Circulator S-parameter measurements at $4.2 \mathrm{~K}$.

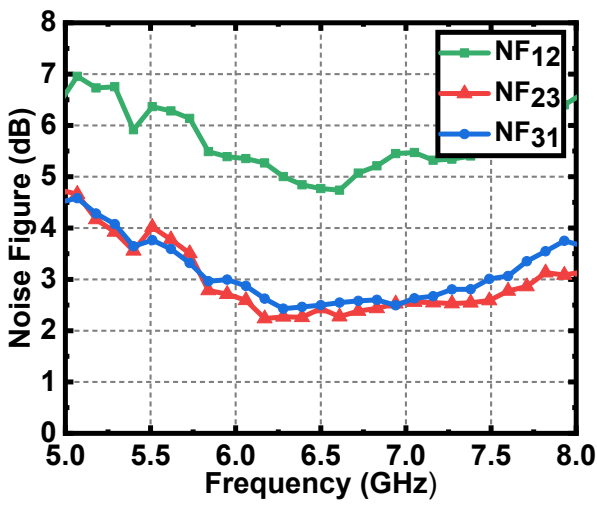

Fig. 8. Circulator noise figure measurements at $300 \mathrm{~K}$.

Noise measurements of the circulator at $300 \mathrm{~K}$ are presented in Fig. 8 and show good agreement with insertion loss results, with a minimum noise figure of $2.4 \mathrm{~dB}$.

The measured circulator IIP3 is higher than $+17.5 \mathrm{dBm}$ in all directions of circulation at both $4.2 \mathrm{~K}$ and $300 \mathrm{~K}$, which is sufficient for quantum computing applications, where the received signal is well below the non-linear region $(-90 \mathrm{dBm})$.

In the target quantum computing application, the proposed circulator is expected to be integrated in a full receiver [3] operating at $4.2 \mathrm{~K}$, so that the clock signal would be generated by the on-chip PLL, thus minimizing noise. Clock feedthrough could be a concern in such application, given the very low-noise requirements and the low signal power levels $(-90 \mathrm{dBm})$. While coupling from clock generation circuitry through the substrate is highly reduced at $4.2 \mathrm{~K}$ thanks to carrier freeze-out, mixer feedthrough may need to be mitigated in the future by a further reduction of the clock frequency or by other techniques, to limit the amplitude of clock harmonics in the circulator band.

Measured results are summarized and compared to stateof-the-art CMOS and commercial circulators in Table 1.

\section{Conclusion}

A 40-nm CMOS circulator has been designed to operate at both $300 \mathrm{~K}$ and $4.2 \mathrm{~K}$ for quantum computing applications.

Thanks to the realization of a large modulation index, enabled by the exploitation of a second-order bridged-T all-pass filter with sharp but linear phase response, power consumption, area and fractional bandwidth are significantly better than prior art.
Table 1. Comparison table with state-of-the-art circulators.

\begin{tabular}{|c|c|c|c|c|c|}
\hline & This work & {$[5]$} & {$[4]$} & {$[6]$} & {$[7]$} \\
\hline Technology & $\begin{array}{c}40-\mathrm{nm} \\
\text { CMOS }\end{array}$ & $\begin{array}{c}65-\mathrm{nm} \\
\text { CMOS }\end{array}$ & $\begin{array}{c}45-\mathrm{nm} \\
\text { SOI }\end{array}$ & $\begin{array}{c}180-\mathrm{nm} \\
\text { SOI }\end{array}$ & Ferrite \\
\hline $\begin{array}{c}\text { Working } \\
\text { temperature (K) }\end{array}$ & $4.2-300$ & 300 & 300 & 300 & $0.02-300$ \\
\hline Architecture & All-pass & N-path & T-line & T-line & Discrete \\
\hline Frequency (GHz) & $5.6-7.4$ & $0.61-0.97^{1}$ & $22.7-27.3$ & $0.86-1.08$ & $4-8$ \\
\hline Modulation index & 5 & 1 & 3 & 3 & N.A. \\
\hline Insertion loss (dB) & 2.2 & 1.7 & 3.2 & 2.1 & 0.4 \\
\hline $\begin{array}{c}\text { Isolation (dB) } \\
\text { Fractional } \\
\text { bandwidth }(\%)\end{array}$ & 18 & $>20$ & 18.5 & $>25$ & 18 \\
\hline Noise figure (dB) & $2.4-3.4$ & 4.3 & $3.3-4.4$ & $2.9-3.1$ & 0.4 \\
\hline IIP3 (dBm) & $>+17.5$ & +27.5 & +20.1 & +50 & N.R. \\
\hline Core area (mm $\left.{ }^{2}\right)$ & 0.45 & 25 & 2.16 & 16.5 & 1575 \\
\hline $\begin{array}{c}\text { Power consumption } \\
(\mathrm{mW})\end{array}$ & $2.5 / 12.4^{3}$ & 59 & 78.4 & 170 & 0 \\
\hline $\begin{array}{c}\text { Normalized power } \\
\mathrm{P}_{\text {DC }} / \mathrm{f}_{0}(\mathrm{~mW} / \mathrm{GHz})\end{array}$ & 1.9 & 75 & 3.1 & 175 & 0 \\
\hline
\end{tabular}

${ }^{1}$ Range of center frequency tunability, ${ }^{2}$ Isolation and 1-dB insertion loss bandwidth, ${ }^{3}$ Core (divider and mixer buffers) power and overall power consumption respectively.

A record-low power consumption of $2.5 \mathrm{~mW}$ and a state-of-the-art fractional bandwidth of $28 \%$ are achieved in only $0.45 \mathrm{~mm}^{2}$ active area. This enables miniaturization and multiplexing of qubit frequencies in power-constrained cryogenic refrigerators. Furthermore, the circulator yields a minimum 2.2-dB insertion loss and 2.4-dB noise figure with $18-\mathrm{dB}$ isolation in the band $5.6 \mathrm{GHz}-7.4 \mathrm{GHz}$.

\section{REFERENCES}

[1] M. F. Gonzalez-Zalba et al., "Probing the limits of gate-based charge sensing," Nature Communications, vol. 6, p. 6084, Jan 2015.

[2] D. Ristè et al., "Deterministic entanglement of superconducting qubits by parity measurement and feedback," Nature, vol. 502, p. 350, Oct 2013.

[3] B. Patra et al., "Cryo-CMOS Circuits and Systems for Quantum Computing Applications," IEEE JSSC, vol. 53, no. 1, pp. 309-321, Jan 2018.

[4] T. Dinc et al., "A Millimeter-Wave Non-Magnetic Passive SOI CMOS Circulator Based on Spatio-Temporal Conductivity Modulation," IEEE JSSC, vol. 52, no. 12, pp. 3276-3292, Dec 2017.

[5] N. Reiskarimian et al., "A CMOS Passive LPTV Non-Magnetic Circulator and Its Application in a Full-Duplex Receiver," IEEE JSSC, vol. 52, no. 5, pp. 1358-1372, May 2017.

[6] A. Nagulu et al., "Fully-Integrated Non-Magnetic 180nm SOI Circulator with $>1 \mathrm{~W}$ P1dB, >+50dBm IIP3 and High Isolation Across 1.85 VSWR," in 2018 IEEE RFIC, June 2018, pp. 104-107.

[7] QuinStar Technology, Inc. website, http://www.quinstar.com. 